\title{
La escala de valoración del estado de ánimo (EVEA): análisis de la estructura factorial y de la capacidad para detectar cambios en estados de ánimo ${ }^{1}$
}

\author{
Tasmania del Pino-Sedeño, Wenceslao Peñate y Juan Manuel Bethencourt \\ Universidad de La Laguna
}

RESUMEN

La Escala de Valoración del Estado de Ánimo [EVEA, Sanz, J. (2001). Análisis y Modificación de Conducta, 27, 71-110] es un instrumento diseñado para evaluar cuatro estados emocionales de carácter situacional con entidad clínica (depresión, ansiedad, hostilidad y alegría). Para el análisis de la fortaleza estructural de la escala, ésta fue administrada a una muestra de 140 participantes. Para conocer la capacidad de la EVEA para monitorizar cambios en el estado de ánimo, 40 de participantes fueron asignados a una tarea de reexperimentación emocional de vivencias traumáticas o a una tarea de escribir sobre hechos triviales. Además de la EVEA, se administró la escala PANAS para evaluar la afectividad positiva y negativa. Los resultados mostraron la consistencia de la escala, su ajuste estructural y su capacidad para detectar los cambios en el estado de ánimo, especialmente en depresión, de manera similar a los detectados por la afectividad negativa del PANAS.

Palabras clave: Escala EVEA, estructura factorial, estados emocionales inducidos.

\section{ABSTRACT}

The Scale for Mood States Assessment [EVEA, Sanz, J. (2001). Análisis y Modificación de Conducta, 27, 71-110] is an instrument designed for measuring transitory moods with a clinical entity (depression, anxiety, hostility and happiness). This scale was administered to a sample of 140 participants. 40 of these participants were assigned to task of disclosure of traumatic emotional experiences or task to write about trivial events, to know the ability to monitor changes in mood. In addition to EVEA, PANAS scale were administered to assess positive and negative affect. The results showed the consistency of the scale, structural adjustment and its ability to detect changes in mood, especially in depression mood state, similar to PANAS negative affectivity.

Key words: EVEA scale, factor structure, induced emotional states. 


\section{Introducción}

La Escala de Valoración del Estado de Ánimo (EVEA, Sanz, 2001) es una escala formada por diferentes adjetivos que valoran cuatro estados de ánimo generales, a saber, depresión, ansiedad, hostilidad y alegría. Es una escala de carácter situacional, elaborada para detectar los cambios que se puedan operar en esos estados producto de cambios ambientales y contextuales.

La necesidad de contar con instrumentos de evaluación autoaplicados que permitan monitorizar los cambios situacionales está a la base misma de la evaluación psicológica y, especialmente, en los modelos de evaluación caracterizados por relacionar directamente la evaluación y el tratamiento psicológico (Peñate, 2008). El modelo conductual de evaluación psicológica tiene como objetivo primordial el llevar a cabo una evaluación que permita una mejor planificación del tratamiento psicológico, dentro de un planteamiento general basado en el análisis funcional de la conducta (Virués-Ortega \& Haynes, 2005).

Evidentemente, la adecuación de esa planificación debe estar contrastada por medio de instrumentos y técnicas que permitan medir los cambios que se van produciendo producto de los cambios inducidos (investigación básica) o la eficacia del programa terapéutico implementado. Usualmente, se han recomendado medidas lo más objetivas posibles (observación directa, registros fisiológicos,...), pero también se ha echado mano de instrumentos que nos den la propia versión de la persona objeto de la evaluación y tratamiento (autorregistros, autoinformes,...). En este sentido, los inventarios y escalas situacionales han jugado un papel relevante en detectar esos cambios. La importancia entonces de contar con instrumentos bien validados es una prioridad de estos modelos de evaluación.

Sin embargo, el interés por evaluar estos estados emocionales no se circunscriben a los modelos conductuales. Desde otras perspectivas (Blanco-Picabía \& Rodríguez Franco, 2008; Elliott, 2010), se ha planteado también la necesidad de contar con instrumentos precisos para evaluar los cambios procesuales, como parte integrante de la eficacia del proceso terapéutico.

Las listas de adjetivos son uno de los instrumentos de evaluación psicológica más conocidos dentro de la evaluación de los estados de ánimo situacionales. Las características de este tipo de instrumentos se ajustan muy bien a las necesidades de la evaluación de los estados de ánimo. Las listas de adjetivo son tareas que proporcionan el máximo de información descriptiva mediante un sistema de respuesta muy sencillo, fiable en su procedimiento de recogida de datos y que requiere muy poco tiempo para su utilización (Ávila \& Giménez, 1991a, 1991b). Además, estos instrumentos permiten aplicaciones repetidas con intervalos breves de tiempo, dada su sensibilidad para reflejar los cambios, y apenas tienden a generar reacciones defensivas de los participantes, ni a activar tendencias de respuesta de forma relevante (Ávila \& Giménez, 1991a). De hecho, las listas de adjetivos es el procedimiento de evaluación de estados de ánimo más utilizado $y$, probablemente, el más adecuado (AndrésPueyo, 1993).

La EVEA representa uno de estos procedimientos para evaluar los cambios en el estado 
de ánimo, apoyado en que evalúa simultáneamente cuatro de ellos. En consonancia con los estados de ánimo más estudiados en la literatura, la EVEA trata de evaluar simultáneamente los estados de depresión, ansiedad, alegría y hostilidad (Sanz, 2001), siguiendo la afirmación de Watson \& Clark (1997): “Recomendamos firmemente que los investigadores del estado de ánimo evalúen de forma rutinaria un amplio rango de estados de ánimo tanto negativos como positivos. ¿Qué estados de ánimo específicos se debería incluir? Ciertamente, una evaluación comprehensiva debería incluir escalas que evalúen el estado de ánimo miedoso/ansioso, triste/deprimido y enfadado/hostil, así como algún tipo de afecto positivo [...]" (p. 200). Por tanto, en la evaluación de procedimientos de inducción de estados de ánimo no parecen tan adecuados los autoinformes que evalúan constructos afectivos globales, pues no permiten una evaluación más específica de diferentes estados de ánimo negativos y positivos.

La EVEA consiste de 16 ítems, por lo que puede aplicarse en menos de dos minutos, cada uno compuesto por una escala tipo Likert de 11 puntos (de 0 a 10) que presenta en su margen izquierdo una corta afirmación que describe un estado de ánimo. Las 16 frases tienen la misma construcción; todas empiezan con las palabras "me siento" y continúan con un adjetivo que representa un estado de ánimo (e.g. "me siento triste", "me siento alegre"). Cada estado de ánimo viene representado por cuatro ítems con diferentes adjetivos, los cuales definen una subescala, de acuerdo con la propuesta metodológica de Nunnally (1987).
Todos los ítems dentro de cada subescala están formulados en la misma dirección.

Para medir los estados de ánimo depresivo y alegre, los adjetivos de la EVEA fueron entresacados de las versiones en español de las Lista de Adjetivos para la Depresión (DACL, Depression Adjetive Check List, Lubin, 1965). De acuerdo con lo que indica el propio autor (Sanz, 2001), los adjetivos para medir el estado de ánimo ansioso fueron seleccionados a partir de los ítems de la escala de estado del State Trait Anxiety Inventory (STAl; Spielberger, Gorsuch, \& Lushene, 1970). Para evaluar el estado de hostilidad, los items fueron extraídos de una traducción que se hizo de las escalas de estado de la Escala de Ira Estado-Rasgo (STAS; Strate-Traig Anger Scale, Spielberger, Jacobs, Russel, \& Crane, 1983). A pesar de algunas limitaciones, en general estos instrumentos son instrumentos bien establecidos y que han demostrado tener unos adecuados índices de fiabilidad y validez.

La investigación sobre los estados de ánimo tiene uno de sus orígenes en los desarrollos teóricos del afecto y la emoción. Las teorías generales del afecto han tratado de identificar los elementos básicos y su configuración a lo largo del ciclo vital (Watson \& Tellegen, 1985).

De acuerdo con Yik, Russell \& Feldman-Barrett (1999), dos serían los elementos comunes a los afectos que, según sea el modelo, se denominarían de una manera u otra. El primero de ellos sería el nivel de activación (arousal, alerta, energía, tensión...). El segundo elemento sería la valencia del afecto (placer, alegría, satisfacción...). Algunos modelos sólo atienden a uno de los elementos, pero es usual que se recurra 
a los dos, combinándolos en una estructura relacional que daría cuenta de los distintos afectos básicos.

Utilizando los dos elementos anteriores (valencia y activación), este modelo sostiene que una estructura ortogonal entre valencia (placer/ displacer) y activación (alta y baja), permitiría identificar y ordenar los afectos básicos. A grandes rasgos, el esquema de los afectos quedaría como se recoge en la Figura 1.

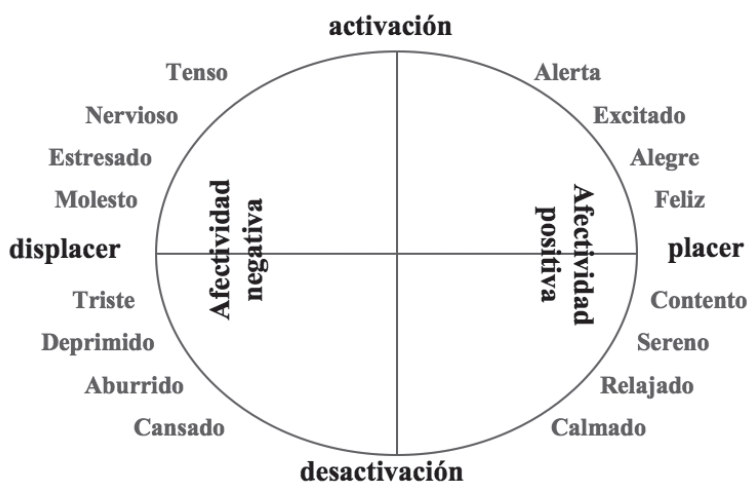

Figura 1. Representación del modelo de afectividad positiva y negativa de Watson \& Tellegen (1985), de acuerdo con la propuesta de ejes ortogonales de Yik et al. (1999).

Como puede observarse, los dos continuos (placer/displacer y activación/desactivación) permiten situar los tipos de afectos y emociones principales. En torno al polo del placer se situarían los afectos que tienen una valencia positiva y en torno al polo del displacer se situarían los afectos de carácter negativo.

Los trabajos que han apoyado la bipolaridad (Crawford \& Henry, 2004; Feldman-Barret \& Russell, 1998; Green, Goldman, \& Salovey, 1993; Larsen \& Diener, 1992), sostienen que la supuesta independencia entre la afectividad positiva y negativa es un problema de error de medida que enmascara una estructura bipolar latente. Por el contrario, los defensores de la tesis de la independencia sostienen que las relaciones negativas y simétricas encontradas en dos polos de los afectos básicos son datos aleatorios (e.g., Watson \& Clark, 1992; 1997; Watson, Wiese, Vaidya, \& Tellegen, 1999). Vautier \& Raufaste (2003), utilizando un modelo de ecuaciones estructurales no encuentran evidencias sólidas ni para la bipolaridad ni para la independencia, cuestionando si los contenidos de los afectos representan a contenidos realmente independientes entre sí.

Los estudios iniciales basados en juicios de similaridad entre palabras, estimaciones derivadas de la utilización del diferencial semántico, sugirieron la existencia de dos o tres dimensiones básicas bipolares (Placer-Displacer, Activación-Inhibición y Atención-Rechazo) (e.g., Bush, 1973). Las investigaciones posteriores, usualmente apoyadas en datos de autoinformes han dado lugar a conclusiones dispares, sugiriendo la existencia de un número amplio de factores (e.g., Izard, 1972). Sin embargo, la mayoría de las propuestas vuelven a la idea inicial de la existencia de dos o tres dimensiones (e.g., Russell \& Mehrabian, 1977). De hecho, los estudios fundamentados en metodologías basadas en técnicas factoriales sugieren que las diferentes dimensiones del afecto se agrupan en dos tipos de factores, uno determinado por un limitado número de factores positivos y otro por un amplio número de factores de afecto negativo (Zevon \& Tellegen, 1982).

En un reanálisis efectuado posteriormente por Watson \& Tellegen (1985) sobre un amplio número de estudios se sugiere que los dos principales factores que aparecen de forma consistente son el afecto positivo y el afecto 
negativo, los cuales configuran fielmente la estructura básica del afecto. En este modelo bifactorial del afecto, denominado modelo PANAS, Watson \& Tellegen (1985) establecen que ambos factores no representan polos dimensionales (como su denominación podría indicar), sino que constituyen dos dimensiones independientes del afecto $y$, por tanto, no correlacionadas. Estas dos dimensiones han sido caracterizadas como "descriptivamente bipolares, pero afectivamente unipolares", para resaltar que solamente el polo alto de cada dimensión representa un estado de alto afecto, siendo el polo bajo de cada factor únicamente un reflejo de ausencia relativa de implicación afectiva. Así por ejemplo, la calma y la relajación serían componentes de bajo afecto negativo, mientras que la desgana y la lentitud/torpeza lo serían de bajo afecto positivo (Watson \& Tellegen, 1985). El afecto positivo (AP) reflejaría a una persona entusiasmada, activa, alerta, con energía y satisfecha. El afecto negativo (AN) representaría una dimensión general de malestar subjetivo y desagradable que incluye una variedad de estados emocionales aversivos como disgusto, ira, culpa, miedo y nerviosismo (Watson, Clark, \& Tellegen, 1988).

La combinación de ambos afectos daría cuenta de la validez diferencial de grupos de afectos más complejos. Así, basado en el modelo PANAS, se ha propuesto el modelo tripartito (Clark \& Watson, 1991). De acuerdo con este modelo, dos trastornos tan comórbidos como la ansiedad y la depresión se caracterizarían por compartir elevados niveles de AN y distinguirse en que la depresión mantendría una relación negativa con la AP (que los pro- ponentes caracterizaron como niveles elevados de anhedonia), mientras que la ansiedad mantendría una relación independiente con la AP (Anderson \& Hope, 2008).

Así, con el objeto de obtener una medida breve y fácil de administrar sobre el afecto positivo y negativo, Watson et al. (1988) construyeron el cuestionario PANAS (Positive and Negative Affect Schedule). Los autores aislaron finalmente 10 marcadores del afecto positivo y 10 para el afecto negativo, los cuales constituyen los 20 ítems de la actual versión de la PANAS. Así pues, mediante el PANAS se podría obtener una puntuación en afectividad positiva (subescala AP) y otra en afectividad negativa (subescala AN).

Los datos psicométricos presentados por Watson et al. (1988) sugieren que ambas subescalas de la PANAS poseen niveles adecuados de fiabilidad y validez (factorial, convergente y discriminante) y presentan evidencia empírica confirmatoria sobre el modelo bifactorial sustentado en las dimensiones AN y AP (Joiner, Sandín, Chorot, Lostao, \& Marquina, 1997; Sandín, Chorot, Lostao, Joiner, Santed, \& Valiente, 1999). Estos datos sugieren que la estructura bidimensional del afecto emerge con independencia del tipo de país y cultura. Asimismo, se ha observado la estructura AN/AP de forma común para los grupos de varones y de mujeres (Sandín, et al. 1999).

Sin embargo, tal y como ha sido propuesto por Yik et al., (1999), todos esos acercamientos, tanto el modelo PANAS como los previamente citados, mantienen entre sí una serie de relaciones que permitirían observar una serie de redundancias y elementos comunes. 
Un paso más lo han dado los estudios de Zautra, Berkhof \& Nicolson (2002) y Davis, Zautra \& Smith (2004). Estos trabajos consideran que los afectos positivo y negativo mantienen relaciones diferentes de acuerdo a la interpretación del contexto que rodea a una persona. Cuando el contexto es valorado como neutral o no amenazante los dos tipos de afectos se mantienen independientes, pero cuando el contexto es valorado como amenazante, tienden a bipolarizarse. De acuerdo con este planteamiento entonces, si modificamos o alteramos el contexto, alteramos la estructura relacional de los afectos básicos. Estos hallazgos, conjuntamente con el cuestionamiento de la independencia o bipolaridad de los afectos básicos, cuestionan y limitan el uso de esos afectos a la hora de determinar los cambios que producen en el estado de ánimo, producto de una inducción o, como hemos señalado, de un programa terapéutico. Si la naturaleza misma de la medida se altera en función de los cambios contextuales, no parecen los mejores marcadores para observar posibles cambios. En este sentido, medidas situacionales menos básicas y más clínicamente asentadas, podrían garantizar una mayor consistencia, a la vez que proporcionarían una medida en relación directa con los cambios que se pretenden generar. En otras palabras, una medida que evaluara situacionalmente condiciones clínicas como la ansiedad, depresión u hostilidad, estaría más vinculada a los cambios que pudiéramos estar intentando generar en esas condiciones.

La escala EVEA, en la medida en que plantea evaluar, no dimensiones básicas del afecto, sino síndromes constituidos por la variabilidad entre esos afectos (ansiedad, depresión, hostilidad...), podría suponer una medida más consistente para evaluar los cambios contextuales y situacionales.

Frente a la potente evidencia mostrada por el modelo PANAS, surge la necesidad tanto en la investigación, especialmente cuando se trata de investigación aplicada, como en la práctica clínica, de medir estados de ánimo de forma más ejemplificada, es decir, se requiere de medidas que no sólo informen de las modificaciones en afectos globales e inespecíficos, sino en estados más concretos como lo son los estados de depresión y ansiedad, para así poder monitorizar los efectos de las distintas intervenciones. Aunque escalas como la PANAS pueden dotar de información veraz sobre cambios en los estados de ánimo, surge la necesidad de especificar concretamente qué es lo que está cambiando y en qué dirección.

En esta investigación en concreto nos interesa conocer la capacidad que posee el EVEA para monitorizar los cambios en el estado de ánimo, detectando los cambios que se producen producto de una sesión de reexperimentación emocional. Inicialmente, debido a que se trata de una escala reciente, se aportarán datos sobre el ajuste estructural/factorial de la escala.

\section{Método Participantes}

Para obtener los datos de este trabajo se partió de una muestra total de 140 participantes, estudiantes en la Universidad de La Laguna, procedentes de estudios en Económicas, Sociología, Pedagogía, Trabajo Social, Logopedia y Psicología. 124 eran mujeres (88,6\% mujeres). La edad media fue de 21,67 años (DT = 
2,84; rango $=19-43$ años). Posteriormente, 40 participantes, fueron asignados a dos condiciones: llevar a cabo una reexperimentación emocional escrita sobre algún suceso traumático o escribir sobre cuestiones triviales triviales. La participación fue voluntaria.

\section{Instrumentos}

Escala de Valoración del Estado de Ánimo, EVEA (Sanz, 2001). Medida anteriormente descrita, es una escala que evalúa los estados emocionales transitorios de ansiedad, depresión, hostilidad y alegría. La EVEA es una lista de adjetivos, elaborada para su utilización en estudios que utilizan procedimientos de inducción de estado de ánimo. Las cuatro subescalas presentan buenos ajustes psicométricos (Sanz, 2001): elevados índices de consistencia interna (coeficiente alfa entre 0,88 y 0,93) buena estabilidad temporal independientes del contexto (correlaciones a los 7 y 25 minutos entre 0,55 y $0,88)$. Así mismo la escala muestra una adecuada estructura factorial (cuatro factores que se corresponden con las subescalas).

Escala de afecto positivo y negativo (PANAS, Watson, et al., 1988) También descrita parcialmente, esta escala contiene 20 descriptores de estados de ánimo (10 positivos y 10 negativo). La validación psicométrica (Watson et al., 1988) ha mostrado la existencia de una estructura bifactorial de afecto positivo (AP) y afecto negativo (AN). Esta estructura bifactorial también se ha verificado en la versión española (Sandín, et al., 1999). En este estudio la PANAS se ha utilizado como criterio de validación de los cambios emocionales.

\section{Diseño}

Se ha utilizado un diseño doble: (i) para la validación de la estructura factorial de la EVEA se ha utilizado un diseño correlacional, contando con la muestra total; y (ii) para la monitorización de los estados de ánimo se ha utilizado un diseño experimental bivariado con un grupo experimental (grupo de la reexperimentación emocional, RE) y un grupo control (escribir sobre cuestiones triviales, GC).

\section{Procedimiento}

La muestra total fue reclutada mediante anuncios en distintas facultades de la Universidad de La Laguna. La participación fue voluntaria y si eran estudiantes de Psicología, se les proporcionaban créditos en una asignatura de la carrera. Si eran estudiantes de otras carreras, se les daba gratuitamente un curso, equivalente a dos créditos dentro de la oferta de libre configuración. A todos ellos se les solicitó la firma del consentimiento informado.

Con la finalidad de inducir un cambio en el estado de ánimo, a 40 de los participantes se les solicitó la colaboración en una investigación sobre reexperimentación emocional, por medio de la escritura de vivencias traumáticas o altamente estresantes. En todos los casos, los participantes fueron evaluados individualmente en salas aisladas de la Facultad de Psicología de La Universidad de La Laguna. Previo al procedimiento de reexperimentación, todos los participantes cumplimentaron las escalas EVEA y PANAS. La mitad de los participantes 
fueron asignados al grupo de RE, cuya tarea era la escritura de vivencias traumáticas o altamente estresantes a través de las instrucciones, derivadas de las instrucciones de Pennebaker (1989): “Los seres humanos estamos expuesto a una serie de hechos que no podemos controlar y que nos suponen un cierto daño psicológico (accidentes de tráfico, desastres naturales, muertes de seres querido, observar la muerte o el suicidio de alguien, ser asaltado, sufrir abusos sexuales, ser violado...). Ahora trata de recordar un hecho que te haya ocurrido o que hubieras presenciado y que te haya resultado altamente molesto, muy estresante o traumático. Preferiblemente céntrate en el que te haya parecido más traumático. Concéntrate en todos los detalles (sonidos, olores, personas, lugares, colores...), cierra los ojos si ello te ayuda a concentrarte. Haz pasar esa historia por tu mente $y$, a continuación, descríbela durante 30 minutos, escribiendo todos los detalles que recuerdes, acompañado de tus sensaciones y tus emociones. Escribe desde lo que sucedió antes del hecho hasta después de pasado el mismo. No te preocupes por la ortografía, la sintaxis o la gramática, solo es interesante que describas tus sentimientos y emociones más profundas asociada a esos hechos".

Por otro lado, la otra mitad de participantes, asignados a la condición de situaciones triviales (GC), respondieron a instrucciones como la siguiente: "Durante 30 minutos, trata de recordar cronológicamente todo lo que has hecho hoy, desde que te levantaste hasta que llegaste aquí. Concéntrate y haz pasar el desarrollo de este día por tu mente $y$, a continuación, escríbelo, describiendo todo los detalles que recuerdes, acompañados de tus sensaciones y tus emociones. No te preocupes por la ortografía, la sintaxis o la gramática".

Ambos procedimientos se llevaron a cabo en salas individuales. Una vez finalizadas las sesiones, se les administraba el EVEA y el PANAS nuevamente.

Los datos fueron grabados y analizados mediante el paquete estadístico SPSS. Se Ilevaron a cabo análisis factoriales, de consistencia interna, correlacionales y contrastes de media (ANOVA).

\section{Resultados}

Un primer análisis se realizó para probar la estructura de la EVEA por medio de un análisis factorial (extracción de los componentes principales de la EVEA con rotación oblicua) sobre los datos de 140 participantes. Se llevó a cabo una rotación oblicua al presumirse relaciones entre los distintos factores. La rotación ofreció una estructura de cuatro factores, con valores propios iguales o superiores a 1,0. En general se observaron saturaciones muy elevadas de los 16 adjetivos en los cuatro factores, con saturaciones secundarias en otros factores. Tomando como criterio saturaciones por encima de 0,75 , se observan cuatro factores claros que representan a las cuatro subescalas de la EVEA (ver Tabla 1). Los factores de Alegría y Hostilidad obtuvieron saturaciones negativas, en todos los ítems, por lo que no se alteró la interpretación de los mismos.

La consistencia interna de estos factores (a de Cronbach) mostraron coeficientes muy altos: Depresión, 0,89; Ansiedad, 0,94; Hostilidad, 0,95, y Alegría, 0,96; favoreciendo con ello 
Tabla 1

Solución factorial de los ítems de la EVEA (extracción ejes principales, rotación oblicua; $n=140$ ).

\begin{tabular}{lcccc}
\hline Ítems de la EVEA & $\begin{array}{c}\text { Factor I } \\
\text { (Depresión) }\end{array}$ & $\begin{array}{c}\text { Factor II } \\
\text { (Ansiedad) }\end{array}$ & $\begin{array}{c}\text { Factor III } \\
\text { (Alegría) }\end{array}$ & $\begin{array}{c}\text { Factor IV } \\
\text { (Hostilidad) }\end{array}$ \\
\hline 4 Melancólico & 0,86 & & & \\
7 Alicaído & 0,90 & & & \\
16 Triste & 0,79 & & & \\
10 Apagado & 0,88 & & & \\
1 Nervioso & & 0,92 & & \\
5 Tenso & 0,92 & & \\
9 Ansioso & 0,94 & & \\
13 Intranquilo & & 0,89 & & \\
15 Contento & & & $-0,96$ & \\
12 Jovial & & $-0,87$ & \\
3 Alegre & & $-0,93$ & $-0,96$ \\
6 Optimista & & & $-0,94$ \\
14 Enfadado & & & $-0,84$ \\
8 Enojado & & & $-0,96$ \\
Irritado & & & \\
11 Molesto & & & \\
\hline
\end{tabular}

Nota: saturaciones mayores que 0,75

la validez estructural de la EVEA en relación con las cuatro subescalas que la componen.

Un tercer tipo de análisis se basó en un análisis correlacional entre los factores de la PANAS y los factores de la EVEA. En la Tabla 2 se resumen los coeficientes encontrados. Como era de esperar de acuerdo con el modelo tripartito, el afecto negativo como afecto genérico de inestabilidad emocional, se relacionó de manera significati- va con todas las variables de la EVEA (negativa con alegría), siendo el coeficiente más elevado con depresión. Con respecto a la afectividad positiva, los coeficientes encontrados fueron negativos (salvo con alegría, el coeficiente más elevado), pero de menor cuantía. Las relaciones con ansiedad y hostilidad no alcanzaron significación estadística. La estructura relacional de las subescalas de la EVEA también mantuvo re-

Tabla 2

Análisis correlacional (Pearson) entre los factores de la EVEA (ansiedad, depresión, hostilidad y alegría) y de la PANAS $(n=140)$.

\begin{tabular}{lcccc}
\hline & AN & ansiedad & depresión & alegría \\
\hline AP & $-0,39\left(^{*}\right)$ & $-0,26$ & $-0,50\left(^{* *}\right)$ & $0,67\left(^{* *}\right)$ \\
AN & & $0,64\left(^{* *}\right)$ & $0,74\left(^{* *}\right)$ & $-0,55\left(^{* *}\right)$ \\
ansiedad & & & $0,59\left(^{* *}\right)$ & $\left.-0,411^{* *}\right)$ \\
depresión & & & $\left.-0,611^{* *}\right)$ \\
hostilidad & & & $-0,48\left(^{* *}\right)$ \\
\hline
\end{tabular}

Nota: $\mathrm{AP}=$ afectividad positivas PANAS; $\mathrm{AN}=$ afectividad negativa PANAS; ${ }^{*} p \leq 0,05 ;{ }^{* *} p \leq 0,01$. 
laciones muy estrechas y significativas entre sí (negativas con alegría).

Para conocer la capacidad de la EVEA para monitorizar los cambios en el estado de ánimo producido por el procedimiento de RE, se llevaron a cabo varios ANOVA, contrastando las puntuaciones del grupo de RE frente al GC. Como medida criterial se utilizó la escala PANAS. Como puede observarse en la Tabla 3, salvo para la subescala de ansiedad (que alcanzó una significación marginal), se observaron diferencias significativas entre el grupo RE y el GC. Estas diferencias fueron en la dirección esperada (más depresión, más hostilidad y menos alegría), en el grupo que llevó a cabo el procedimiento de recuperar la vivencia de un hecho traumático o muy doloroso. Como era de esperar, también mostraron mayor afectividad negativa y menor afectividad positiva, medidas por la escala PANAS.

Con respecto al alcance, en la figura 2 se representan los tamaños del efecto encontrados.

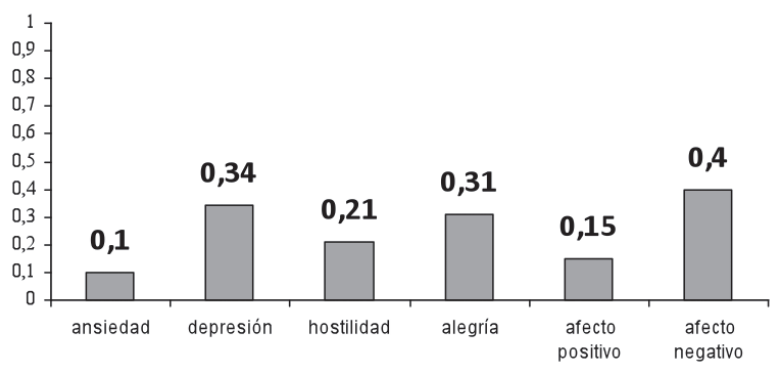

Figura 2. Coeficientes del tamaño del efecto (eta cuadrado) de los contrastes entre los grupos de reexperimentación emocional y grupo control en las variables evaluadas por la EVEA y la PANAS.

Como puede observarse, los valores de $\eta^{2}$ (entendidos como porcentajes de varianza explicados), las escalas que mayor varianza explicaron (por encima de un 30\%) fueron las de afectividad negativa, la depresión y la escala de alegría. La subescala de ansiedad (y la de afectividad positiva), fue la que menos se logró movilizar con el procedimiento de RE.

Tabla 3

Contrastes de medias entre el grupo RE y el GC en las escalas de la EVEA (ansiedad, depresión, hostilidad y alegría) y de la PANAS $(n=40)$.

\begin{tabular}{lccccc}
\hline Variables & Grupo & $M$ & $D T$ & $F$ & $p$ \\
\hline \multirow{2}{*}{ Ansiedad } & RE & 19,37 & 10,98 & 3,66 & 0,064 \\
& GC & 12,41 & 10,79 & & \\
Depresión & RE & 20,21 & 12,60 & 17,85 & 0,001 \\
Hostilidad & GC & 6,00 & 6,09 & & \\
& RE & 12,68 & 9,92 & 8,88 & 0,005 \\
Alegría & GC & 4,06 & 7,00 & & 0,001 \\
& RE & 14,37 & 11,16 & 14,97 & \\
AP & GC & 26,88 & 7,71 & & 0,022 \\
AN & RE & 13,95 & 6,66 & 5,79 & \\
& GC & 19,06 & 6,00 & & 0,001 \\
\hline
\end{tabular}

Nota: $\mathrm{AP}=$ afectividad positivas PANAS; $\mathrm{AN}=$ afectividad negativa PANAS; $\mathrm{RE}=$ reexperimentación emocional; $\mathrm{GC}=$ grupo control; $M=$ media: $D T=$ desviación típica; $p=$ probabilidad. 


\section{Discusión y conclusiones}

Los resultados obtenidos hasta aquí apoyan empíricamente la estructura factorial teorizada por Sanz (2001) y reproducen las cuatro subescalas de la EVEA: depresión, ansiedad, hostilidad y alegría. Cada uno de los ítems del EVEA satura de manera elevada en cada subescala correspondiente, mostrando niveles también elevados de consistencia interna.

Las relaciones entre las escalas denotan la complejidad que supone evaluar emociones complejas, con frecuencia estrechamente relacionadas entre sí. Así, la conocida comorbilidad entre ansiedad y depresión se constata con relaciones muy elevadas entre las dos subescalas (con esperables relaciones negativas con la subescala de alegría). La subescala de hostilidad se comporta de manera similar, relacionándose estrechamente con las otras escalas. Por los contenidos, esta subescala, conceptualmente más compleja, se situaría afectivamente en el displacer activo, similar a la ansiedad. Por otro lado, la irritabilidad depresiva guarda estrechas relaciones con la hostilidad como elemento actitudinal, lo que justificaría también las relaciones estrechas con la subescala de depresión.

La mayor dificultad se encuentra en las saturaciones secundarias que los ítems/adjetivos de una escala poseen en las otras subescalas, lo que podría estar indicando que la totalidad de la escala sería más una medida de malestar psicológico general. Sin embargo, las relaciones con a la afectividad negativa y positiva parecen justificar la presencia al menos de las subescalas de ansiedad y depresión. Tal y como había predicho el modelo tripartito (Clark \& Watson,
1991), la AN se relaciona de manera significativa con la ansiedad y la depresión (también con hostilidad). La AP, en cambio, mantiene relaciones negativas con ambas escalas, aunque sólo adquiere significación para la depresión. Más allá de suponer un cierto apoyo a un modelo tan consolidado como el tripartito (la AN es común a ansiedad y depresión, y la AP es independiente de la ansiedad y mantiene relaciones negativas con depresión), también supone diferenciar entre ambas subescalas, evitando su cuestionamiento a favor de una interpretación más global de la EVEA.

Otro problema podría estribar en los niveles elevados de consistencia interna, con tres escalas con niveles iguales o superiores a 0,94. Estos niveles podrían indicar que los cuatro adjetivos que miden cada una de estas subescalas son psicológicamente muy similares, por lo que, más que complementarios, serían intercambiables entre sí. En este sentido, podría apelarse a los que sostienen que este tipo de medidas muy genéricas se podrían evaluar con uno o dos ítems. Así, Kroenke, Spitzer \&WiIliams (2003), sostienen que serían suficientes dos ítems para detectar el hecho depresivo. Evidentemente este es una controversia que habría que aclarar. Pero, en todo caso, la EVEA es ya una escala muy sencilla, que se administra rápidamente, por lo que la disminución del número de ítems no supondría una mejora apreciable, mientras que dejarla como está permite cumplir con algunos requisitos metodológicos para este tipo de medidas (Nunnally, 1987).

El aspecto más importante de la escala, no obstante, tenía que ver con su capacidad para detectar los cambios inducidos contextual- 
mente. Se esperaba que una escala de carácter situacional permitiera observar los cambios inducidos también de manera situacional. La escala EVEA se ha mostrado como una medida sensible a los cambios transitorios de estados de ánimo con el procedimiento de reexperimentar por un periodo corto de tiempo algún hecho traumático, estresante o altamente molesto: La variable depresión muestra una capacidad similar a una medida de malestar general como es la afectividad negativa. El resto de medidas muestran también su capacidad para detectar los cambios introducidos, salvo la ansiedad, no encontrándose diferencias entre los que llevaron a cabo la reexperimentación y los que no lo hicieron. Es probable que este tipo de recuerdo deprima más que active, como se ha revelado en otros trabajos (Peñate, del Pino-Sedeño \& Bethencourt, 2010), y posiblemente tenga más relación con la incapacidad del procedimiento de reexperimentación para modificar esta variable que con la sensibilidad de la escala.

En definitiva, la escala EVEA muestra una estructura factorial robusta, obteniéndose una conformación de acuerdo con las cuatro variables previstas. Las relaciones con las medidas generales del afecto, permiten apreciar diferencias entre esas cuatro variables, especialmente entre la ansiedad y la depresión. Las elevadas consistencias internas de las subescalas podrían plantear la posibilidad de reducir aún más la composición de los ítems/ adjetivos, pero, debido a la rapidez y sencillez actual, la disminución no supondría un cambio cualitativo en su aplicación. Por otro lado, las cuatro escalas permiten apreciar de manera diferencial los cambios emocionales inducidos. En este caso un procedimiento de RE ha generado cambios en depresión, alegría y hostilidad, pero no en ansiedad. Esta sensibilidad diferencial no se observa en el caso de medidas generales del afecto, favoreciendo con ello la validez incremental de contar con medidas más específicas, frente a medidas globales y sin una clara significación clínica.

\section{Bibliografía}

Anderson, E. R., \& Hope, D. A. (2008). A review of the tripartite model for understanding the link between anxiety and depression in youth. Clinical Psychology Review, 28, 275287.

Andrés-Pueyo, A. (1993). Evaluación del estado de ánimo y de la activación: estado de la cuestión. En M. Forns y M. T. Anguera (Eds.), Aportaciones recientes a la evaluación psicológica (pp. 283-306). Barcelona: PPU.

Ávila, A. \& Giménez, A., (1991a). Los adjetivos en tareas de evaluación psicológica: Propiedades y valor estimular. Revista de Psicología General y Aplicada, 44, 465-475.

Ávila, A. \& Giménez, A., (1991b). Adjectives in psychological assessment tasks: Instruments and applications in the psychology of emotion and personality. Psychological Assessment, 7, 307-331.

Feldman Barrett, L. F., \& Russell, J. A. (1998). Independence and bipolarity in the structure of affect. Journal of Personality and Social Psychology, 74, 967-984.

Blanco-Picabía, A., \& Rodríguez-Franco, L. (2008). La Psicología Clínica: una reflexión. Análisis y Modificación de Conducta, 34, 291304.

Bush, L. E. (1973). Individual differences multidimensional scaling of adjectives denoting feelings. Journal of Personality and Social Psychology, 25, 50-57. 
Clark, L. A., \& Watson, D. (1991). Tripartite model of anxiety and depression: Psychometric evidence and taxonomic implications. Journal of Abnormal Psychology, 100, 316-336.

Crawford, J. R., \& Henry, J. D. (2004). The Positive and Negative Affect Schedule (PANAS): Construct validity, measurement properties and normative data in a large non-clinical sample. British Journal of Clinical Psychology, 43, 245-265.

Davis, M. C., Zautra, A. J., \& Smith, B. W. (2004). Chronic pain, stress, and the dynamics of affective differentiation. Journal of Personality, 72, 1133-1159.

Elliott, R. (2010). Psychotherapy change process research: realizing the promise. Psychotherapy Research, 20, 123-135.

Green, D. P., Goldman, S. L., \& Salovey, P. (1993). Measurement error masks bipolarity in affect ratings. Journal of Personality and Social Psychology, 64, 1029-1041.

Izard, C. E. (1972). Patterns of emotions. New York: Academic Press.

Joiner, Jr., T. E., Sandín, B., Chorot, P., Lostao, L., \& Marquina, G. (1997). Development and factor analytic validation of the SPANAS among women in Spain: (More) Crosscultural convergence in the structure of mood. Journal of Personality Assessment, 68, 600-615.

Kroenke, K., Spitzer, R. L., \& Williams, J. B. (2003). The Patient Health Questionnaire-2: validity of a two-item depression screener. Medical Care, 41, 1284-1292.

Larsen R. J., \& Diener, E. (1992). Promises and problems with circumplex model of emotion. En M. S. Clark (Ed.), Review of personality and social psychology (pp. 2529). Newbury Park, CA: Sage.

Lubin, B. (1965). Adjective check lists for measurement of depression. Archives of General Psychiatry, 12, 57-62.

Nunnally, J. C. (1987) Psychometric Theory. New York: McGraw-Hill.
Pennebaker, J. W. (1989). Confession, inhibition, and disease. En L. Berkowitz (Ed.). Advances in experimental social psychology, vol. 22 (pp. 211-244). San Diego, CA: Academic Press.

Peñate, W. (2008). Psicología clínica y psicodiagnóstico: sobre la vigencia del modelo de evaluación conductual. Análisis y Modificación de Conducta, 34, 95-125.

Peñate, W., del Pino-Sedeño, T., \& Bethencourt, J. M. (2010). El efecto diferencial de escribir sobre un mismo trauma o sobre diferentes traumas en el bienestar psicológico y el estado de salud autoinformado. Universitas Psychologica, 9, 433-445.

Russell, J. A., \& Mehrabian, A. (1977). Evidence for a three-factor theory of emotions. Journal of Research in Personality, 11, 273294.

Sandín, B., Chorot, P., Lostao, L., Joiner, T. E., Santed, M. A., \& Valiente, R. M. (1999). Escala PANAS de afecto positivo y negativo: validación factorial y convergencia transcultural. Psicothema, 11, 37-51.

Sanz, J. (2001). Un instrumento para evaluar la eficacia de los procedimientos de inducción de estado de ánimo: "La Escala de Valoración del Estado de Ánimo" (EVEA). Análisis y modificación de conducta, 27, 71110.

Spielberger, C. D., Gorsuch, R. L., \& Lushene, R. E. (1970). Manual for the State-Trait Anxiety Inventory. Palo Alto, CA: Consulting Psychologist Press (Adaptación española,

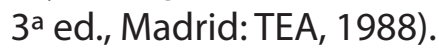

Spielberger, C. D., Jacobs, G., Russel, S., \& Crane, R. S. (1983). Assessment of anger:The StateTrait Anger Scale. En J. N. Butcher and C. D. Spielberg (Eds.), Advances in personality assessment, vol. 2 (pp. 159-187). Hillsdale, NJ: LEA.

Vautier, S., \& Raufaste, E. (2003). Measuring dynamic bipolarity in positive and negative activation. Assessment, 10, 49-55. 
Virués-Ortega, J., \& Haynes, S. (2005). Functional analysis in behavior therapy: Behavioral foundations and clinical application. International Journal of Clinical and Health Psychology, 5, 567-587.

Watson D., \& Clark L. A. (1992). On traits and temperament: general and specific factors of emotional experience and their relation to the five-factor model. Journal of Personality, 60, 441-476.

Watson D., \& Clark L. A. (1997). Measurement and mismeasurement of mood: recurrent and emergent issues. Journal of Personality Assessment, 68, 267-296.

Watson, D., Clark, L. A., \& Tellegen, A. (1984). Cross-cultural convergence in the structure of mood: A Japanese replication and comparison with U. S. findings. Journal of Personality and Social Psychology, 47, 127144.

Watson D., Clark L. A., \& Tellegen A. (1988). Development and validation of brief measures of positive and negative affect: the PANAS scales. Review of personality and social psychology, 54, 1063-1070.

Watson D., \& Tellegen A. (1985). Toward a consensual structure of mood. Psychological Bulletin, 98, 219-335.

Watson, D., Wiese, D., Vaidya, J., \& Tellegen, A. (1999). The two general activation systems of affect: Structural findings, evolutionary considerations, and psychobiological evidence. Journal of Personality and Social Psychology, 76, 820-838.

Yik, M. S. M., Russell, J. A., \& Feldman Barrett, L. F. (1999). Structure of selfreported current affect: Integration and beyond. Journal of Personality and Social Psychology, 77, 600-619.

Zautra, A. J., Berkhof, J., \& Nicolson, N. A. (2002). Changes in affect interrelations as a function of stressful events. Cognition and Emotion, 16, 309-318.
Zevon, M. A., \& Tellegen, A. (1982). The structure of mood change: An idiographic/ nomothetic analysis. Journal of Personality and Social Psychology, 43, 111-122. 